\title{
Additive Technologies in the Production of Vehicle Rubber with Sensory Properties
}

\author{
Evgeniy V. Stepanov ${ }^{1, a *}$, Marek Schmidt ${ }^{2, b}$, Dennis Bäcker ${ }^{2}$ and \\ Anton V. Tumasov ${ }^{1}$ \\ ${ }^{1}$ Nizhny Novgorod State Technical University n.a. R.E. Alekseev, st. Minina, 24, 603950 Nizhny \\ Novgorod, Russia
}

${ }^{2}$ Chemnitz University of Technology, Straße der Nationen 62, 09111 Chemnitz, Germany

aevgeniystepanov1991@nntu.ru, b marek.schmidt@mb.tu-chemnitz.de

\begin{abstract}
Keywords: Sensor Integration, Rubber with Sensory Properties, Piezoceramics, Vehicle, Additive Technologies
\end{abstract}

\begin{abstract}
Annotation. Rubber products are widely used in the construction of vehicles, for example, as sealing and protective devices, suspension joints and are the basis of automobile tires. Modern trends related to increasing the level of vehicle safety require the use of innovative approaches in the design and use of new materials with unique properties. This article proposes an approach to create a rubber with sensory properties that can be used in various automotive products and prevent situations that can harm both human health and lead to serious damage to the structure of the vehicle itself. We have developed an intelligent vehicle door seal to prevent injury to a person when the door is closed carelessly. The sealant, which reacts to deformation when a foreign body enters the seal site, consists of rubber with the addition of piezoceramic powder and two electrode layers. Each electrode layer has several parallel strip-like electrodes positioned along the perimeter of the seal. This document describes possible applications for rubber products with sensory properties and an additive method for making such rubber with the addition of piezoceramic powder.
\end{abstract}

\section{Introduction}

The creation of an intelligent and safe vehicle is an urgent task. A person getting injured when using a vehicle can be not only in a road accident, but also in normal situations related to the embarkation / disembarkation of passengers or loading / unloading a vehicle. Children are often injured when they close the vehicle door on their own themselves. To prevent such situations, this article proposes the use of an intelligent rubber door seal with sensory properties.

Another possible application of smart rubber to improve safety is to use this approach in the manufacture of automobile tires. A large number of accidents are associated with the destruction of tires when the car is moving, the consequences of such situations, as a rule, become critical for maintaining safe movement. The creation of an intelligent bus will allow monitoring the state of its structure and the loads on it during operation. If a defect in the structure or tire overload, which can lead to a critical situation, is detected, it becomes possible to inform the driver about the danger or prevent movement by turning the vehicle into autonomous mode (for example, for self-driving vehicles or vehicles equipped with ADAS).

Currently, there are various additive methods for the manufacture of thermoplastic elastomers [1]. However, for the additive manufacturing of vulcanizable rubber, no commercially viable solution is available on the market. Only a few research approaches can be found in the literature. This is caused by the rheological material behavior of vulcanizable rubber as well as the following 
vulcanization process. This leads to specific challenges for applications related to additive manufacturing processes [2]. In particular, the viscosity of rubber compounds leads to the risk that components built up in layers will deform unintentionally above a certain layer height or collapse completely under their own weight. One possible solution is the parallel layer-by-layer build-up of an additional material that completely encloses and thus supports the rubber shape. The enclosing material also serves to stabilize the shape during the vulcanization process. As additional material for dimensional stabilization of the rubber shape there are investigations with thermoplastic material [3, 4] as well as molding sand, silicone rubber and plaster [5]. However, the above approaches are still subject of research and are not mature processes. An alternative variant is to design the rubber compounds so that self-vulcanization takes place when they are combined. During the additive manufacturing process, the materials are mixed together in a screw extruder and discharged in layers via a nozzle. Subsequently, the vulcanization begins on the printing platform, which keeps the geometrically desired shape stable [6]. However, the possible properties of the finished part remain very limited due to the predominantly predetermined chemical composition of the rubber compounds.

This article proposes to use methods of additive production of vulcanizable rubbers to create a flat composite intelligent material by adding a piezoceramic component to the composition. In 3D printing, rubber and piezoceramic powder is fed into the machine's extruder, which mixes the structures before the resulting material leaves the nozzle. Then, vulcanization of the composite rubber can be done in an oven. At the intermediate stage of 3D printing, it is possible to integrate electrode layers into the sensor rubber to obtain a signal upon deformation of the product, which is difficult to implement with other manufacturing methods. The rubber composite additive machine uses a screw extruder, which gives more flexibility in the shape of the feed material. The material can be used, for example, in the form of powder, granules, liquid or strips. In particular, extrusion-based 3D printing is already being used for non-thermoplastic materials such as ceramics [7] or composites [8].

The aim of this study is to study the technological process of $3 \mathrm{D}$ printing rubber with the addition of ceramic powder based on extrusion and testing a prototype rubber with sensory properties using the example of a vehicle door seal.

\section{The structure of piezoceramic rubber and the principle of its manufacture}

Figure 1 shows the schematic structure of a piezoceramic rubber. The rubber material contains piezoceramic particles inside. On the outside, the rubber is covered with a flexible electrode layer. When the rubber is deformed, pressure is applied to the piezoceramic particles and the direct piezoelectric effect takes place, resulting in a measurable electrical signal. The sensitivity of the signal depends on the amount and distribution of particles in the rubber. Due to the resilience of the rubber, the sensitivity generally does not come close to existing stiff sensors. However, stiff sensors are not designed for integration into rubber parts.
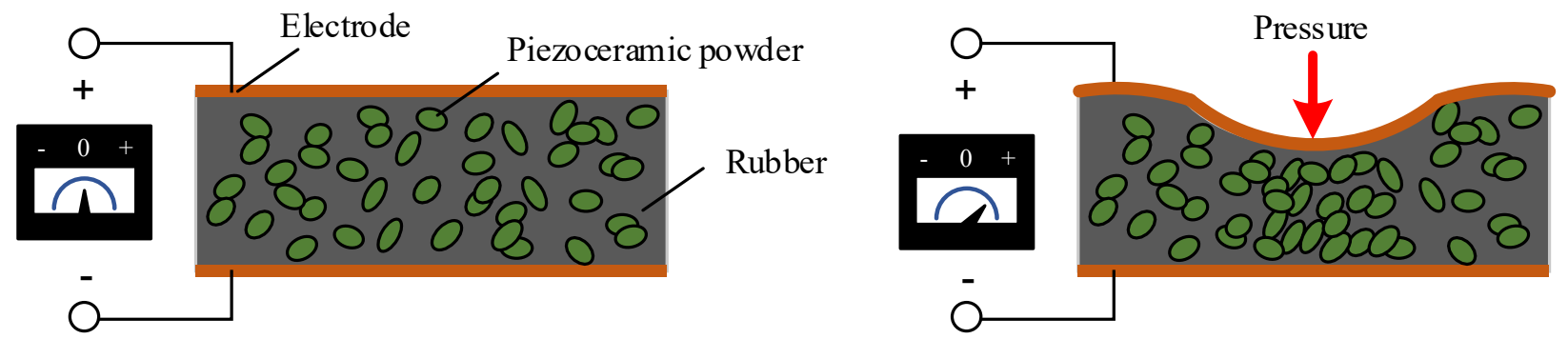

Fig. 1. Operating principle of a piezoceramic rubber 
As described above, the biggest challenge for the additive manufacturing of vulcanizable rubbers is the maintenance of the shape during printing and vulcanization. Nevertheless, flat twodimensional geometries can already be produced without support material [2]. The basis of the technological equipment for the production of composite rubber compounds is the FDM printer (see Figure 2). The printer had to be modified with a screw extruder, as is usually the case when processing rubbers by injection molding. The viscosity of the printing materials plays a particularly important role for the print quality. Nitrile butadiene rubber (NBR) with its viscosity at a printing temperature of $70^{\circ} \mathrm{C}$ has proven to be well suited [2] and was used for the printing tests. Piezoceramics in the form of powder were mixed into the rubber compound to create the sensory composite material.

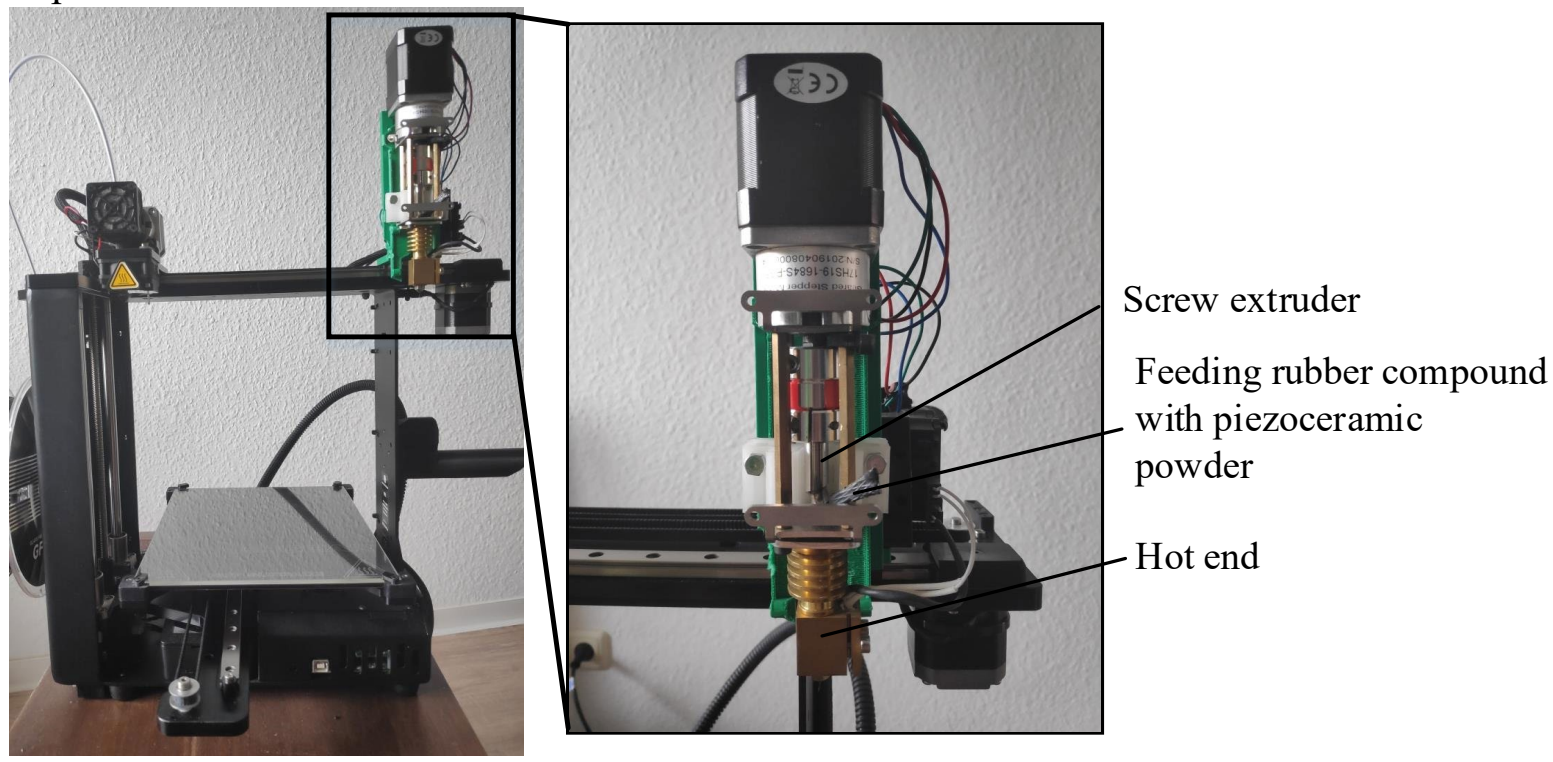

Fig. 2. The MAKERGEAR M3-ID desktop 3D printer modified with a screw extruder

A prototype sealant was printed on the FDM printer while feeding material into the screw extruder. Figure 3 a presents first trial of printing rubber with piezoceramic powder. It can be seen that the piezoelectric component is not homogeneously distributed throughout the compound during the printing process. This process requires a significant improvement. Furthermore, it could be shown in further printing tests, that the printing on a metallic material (brass foil) is possible with an adhesive. This offers the possibility of attaching one of the two necessary electrodes in this way.

a)

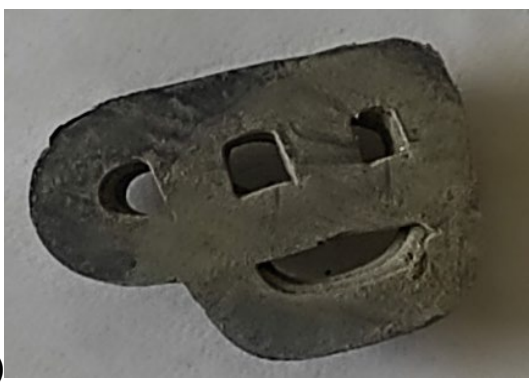

b)

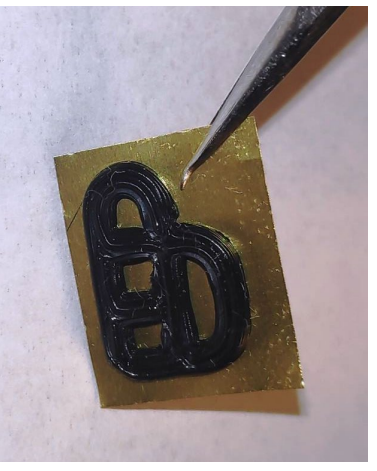

Fig. 3. a) Printed prototype sealant with rubber/piezoceramic powder mixture, b) Printed prototype sealant on a brass foil with an adhesive 


\section{Application of piezoceramic rubber in vehicle}

A general view of the intelligent door seal system is shown in Figure 4. The microcontroller (item 1) is highly resistant to electromagnetic interference and vibration to be installed in a door or on a car body. This microcontroller (printed circuit board) uses a simple method to measure the current [9] flowing through the piezoceramic seal (item 2) when it is deformed. The sensory rubber used for an intelligent door seal must not only have the required elasticity to provide a sealing function, but also be highly sensitive to local deformation to detect foreign bodies around the perimeter of the car door. To prevent the door from closing completely (item 4) when a foreign body is detected at the junction with the body, an electromechanical actuator (item 3) is used, which is embedded in the swing mechanism and is kinematically connected to the body and the door. When a control signal is supplied from microcontroller 1, the actuator switches from passive mode to active mode and instantly creates a braking force that prevents further movement of the car door. This ensures the process of safely closing the door without causing injury to a person and no damage to the car body.

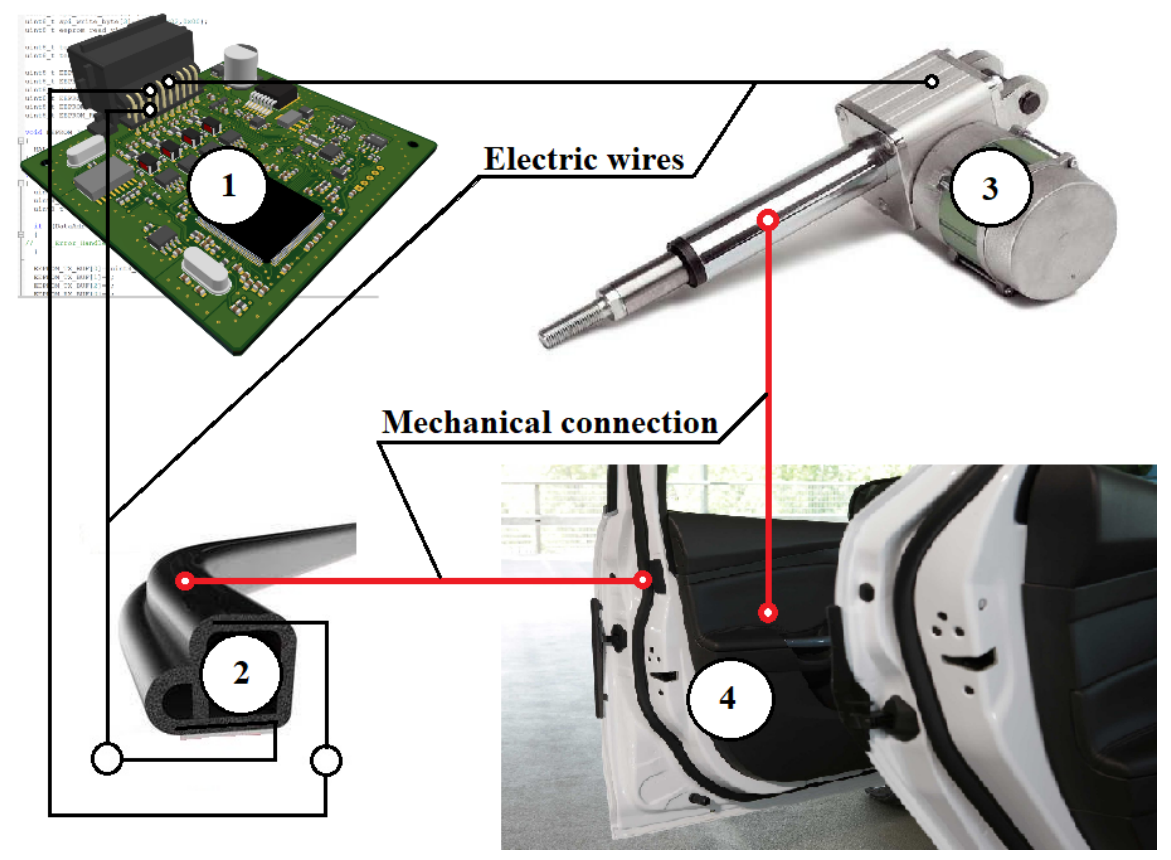

Fig. 4. Schematic diagram and composition of the intelligent door seal system

The system for monitoring the condition of automobile tires is shown in Figure 5. This system also has a microcontroller (item 1) that uses the method of measuring the current flowing through the piezoceramic layer of the tire (item 2) when it is critically deformed or damaged. The rubber with sensory properties used for a smart tire must have the required elasticity, high durability and low sensitivity to small deformations of the tire, allowing it to filter minor loads that cannot lead to damage to the tire structure. 


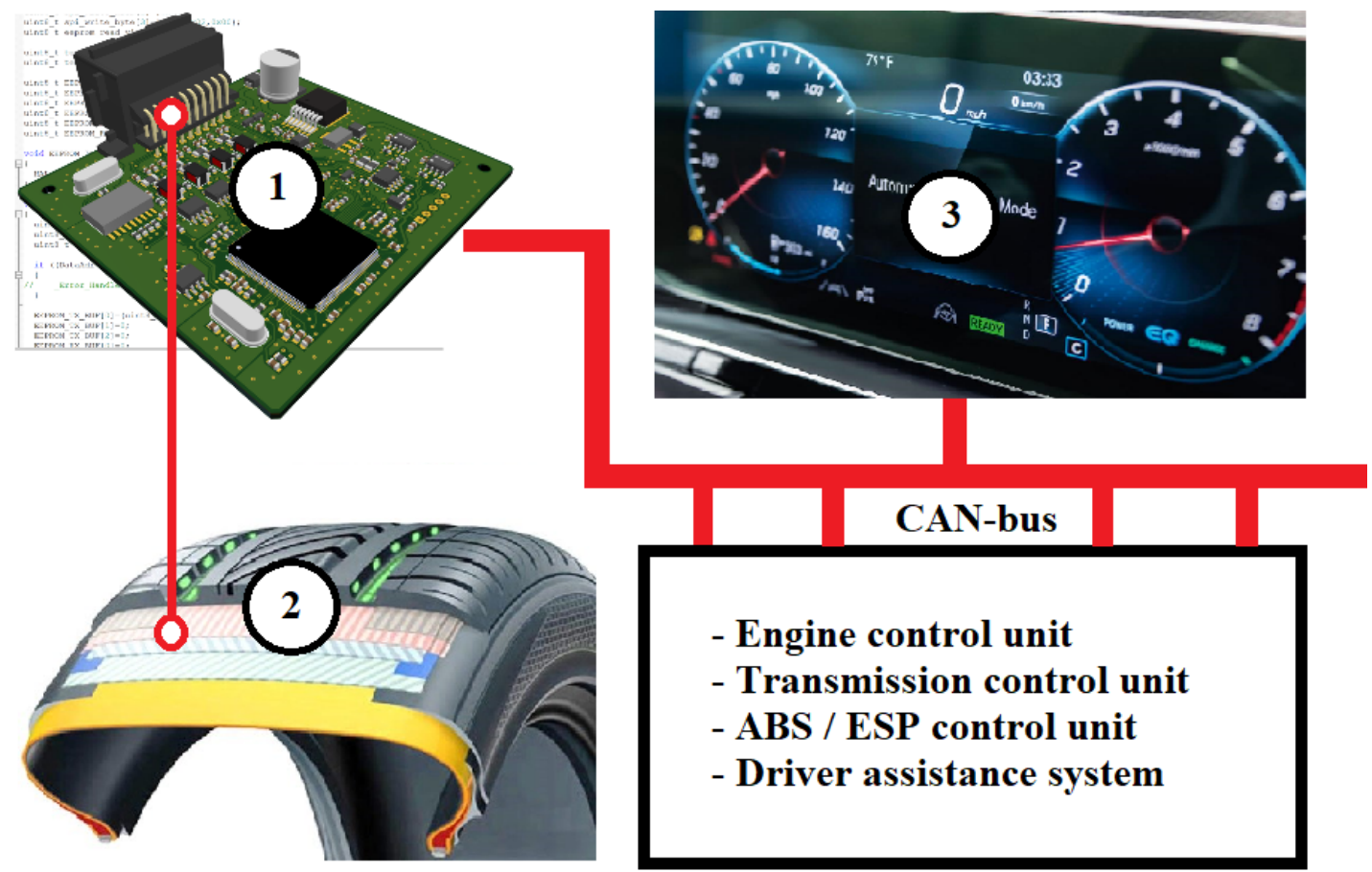

Fig. 5. Schematic diagram and composition of the intelligent vehicle tire system

\section{Signal testing of piezoceramic rubber}

The measurements are associated with many technical difficulties. The additive manufacturing has not yet been able to achieve uniform distribution of the piezoceramic powder (see Figure 3a). Therefore, many small samples ca. $10 \mathrm{~mm} \times 7 \mathrm{~mm}$ (Figure 6) with different proportion of piezoelectric material were prepared by hand. If the ceramic content is very high, the rubber in case of strong deformation tends to easy break at the bending point. Unfortunately, at this stage, a reasonable electrical signal can only be detected with such samples. In the case of samples with a significantly lower proportion of ceramic, the measured signal is not discern at all or hardly distinguishable from the noise.

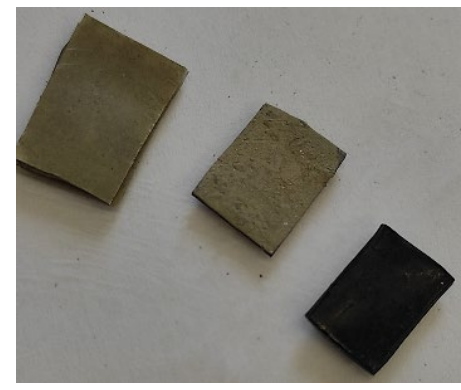

Fig. 6. Two test samples on the left side with piezoelectric material content without electrodes in comparison to the original rubber (right sample).

The Figure 7 shows the measured electrical signal during bending tests. It is obvious that the signal has a strong noise component. Nevertheless, two bending tests with approx. $15 \mathrm{mV}$ and 20 $\mathrm{mV}$ can be identified. 


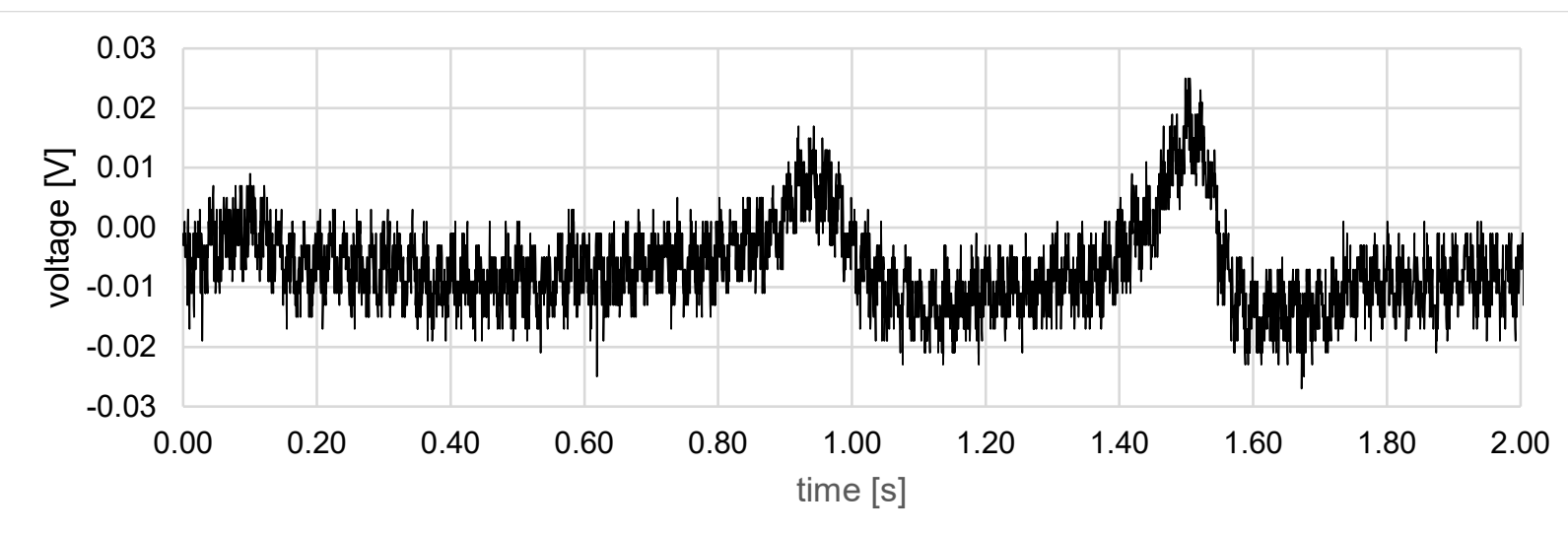

Fig. 7. Electrical signal measured during strong deformation of piezoelectric rubber.

Another difficulty lies in sufficient contacting of the surface. A simple variant of the electrode is the electrically conductive silver layer. The very thin layer due to insufficient adhesion flakes off the surface after a few bending attempts and contributes to the strong noise during the measurement. Therefore, contacting is still an important subject of investigation.

\section{Conclusions}

The results of this study open up a new perspective for the application of smart rubbers in automotive designs. The use of such a composite material is possible not only to improve safety for new cars, but can also serve as an indicator at various stages of the manufacturing process of rubber products. For example, in the manufacture of automobile tires, it is possible to trace the presence of defects in the finished product when compared to a reference tire using non-destructive testing methods by analyzing signals from the smart bus.

Given these circumstances, the present study points to the feasibility of realizing the use of the intelligent automotive door seal and intelligent tire as a commercial product for automotive rubber structures. In addition, the rubber functions implemented in this article can also be useful outside of the automotive industry.

\section{References}

[1] Cohen, D.; Sargeant, M.; Somers, K. 3-D printing takes shape. McKinsey Q. 2014, 1, 1-6.

[2] Drossel, G.-W.; Ihlemann, J.; Landgraf, R.; Oelsch, E.; Schmidt, M. Basic Research for Additive Manufacturing of Rubber.Polymers2020,12, 2266.

https://doi.org/10.3390/polym12102266

[3] Wittek, H.; Klie, B.; Giese, U.; Kleinert, S.; Bindszus, L.; Overmeyer, L. Approach for additive Manufacturing of high-viscosity, curable Rubbers by AME Processing (Additive Manufacturing of Elastomers) — Rubber 3D.KGK Rubberpoint Mach. Euipments, 2019,10, 3035

[4] Sundermann, L.; Klie, B.; Giese, Z.; Leineweber, S.; Overmeyer, L. Development, Construction and Testing of a 3D-Printing-Systemfor Additive Manufacturing of Carbon Black filled Rubber Compounds.KGK Rubberpoint Mach. Euipments2020,6, 53-56.

[5] Drossel, G.-W.; Ihlemann, J.; Landgraf, R.; Oelsch, E.; Schmidt, M. Media for Dimensional Stabilization of Rubber Compounds during Additive Manufacturing and Vulcanization.Polymers2020,12, 2266. https://doi.org/10.3390/polym12102266 
[6] Scheungraber, P. Apparatus and Method for 3D Printing a Workpiece of a Rubbery NonThermoplastic. Material. Patent No. DE102017207737A1, 8 November 2018.

[7] Faes, M.; Valkenaers, H.; Vogeler, F.; Vleugels, J.; Ferraris, E. Extrusion-Based 3D printing of ceramic components. Procedia CIRP 2015, 28, 76-81.

https://doi.org/10.3390/polym12102266

[8] Buswell, R.A.; Leal de Silva, W.R.; Jones, S.Z.; Dirrenberger, J. 3D printing using concrete extrusion: A roadmap for research. Cem. Concr. Res. 2018, 112, 37-49.

https://doi.org/10.1016/j.cemconres.2018.05.006

[9] Reif, K., Ed. Automobilelektronik. Eine Einführung für Ingenieure; Vieweg und Teubner:

Wiesbaden, Germany, 2007. 